\title{
APLICACIÓN DE HIDRÓXIDOS DOBLES LAMINARES DE ZnCUAI MODIFICADOS CON SDS COMO FOTOCATALIZADORES PARA EL TRATAMIENTO EFICIENTE DE AGUA CONTAMINADA CON COMPUESTOS ORGÁNICOS
}

\section{Guadalupe Romero-Ortiz ${ }^{1}$, Enrique Samaniego-Benítez ${ }^{2}$,}

Luis Lartundo-Rojas ${ }^{3}$, Yolanda Flores-Jiménez ${ }^{1,4}$, Francisco Tzompantzi, Angeles Mantilla ${ }^{1 *}$

${ }^{1}$ Instituto Politécnico Nacional, Laboratorio de FOTOCATÁLISIS, CICATA-Legaria, Legaria 694, México. CDMx.

${ }^{2}$ Cátedras CONACyT, Instituto Politécnico Nacional, Laboratorio de FOTOCATÁLISIS, CICATA-Legaria, Legaria 694, México. CDMx.

${ }^{3}$ Instituto Politécnico Nacional, Centro de Nanociencias y Micro y Nanotecnologías, Luis Enrique Erro s/n, Zacatenco, México. CDMx.

${ }^{4}$ Depto. de Química, Área de Catálisis, Grupo ECOCATAL, UAM-Iztapalapa, Av. San Rafael Atlixco 186, México. CDMx.

tomitzi_21@hotmail.com, enriquesabe1809@gmail.com, llartundo@ipn.mx,yolandajimenezflores@gmail.com, fjtz@xanum.uam.mx, angelesmantilla@yahoo.com.mx https://doi.org/10.3926/oms.401.4.2 
118 AVANCES DE INVESTIGACIÓN EN NANOCIENCIAS, MICRO Y NANOTECNOLOGÍAS

Romero Ortíz, G., Samaniego Benítez, E., Lartundo Rojas, L., Jimenez Flores, Y., Tzompantzi, F., \& Mantilla, A. (2020). Aplicación de Hidróxidos dobles laminares de $\mathrm{ZnCuAl}$ modificados con SDS como fotocatalizadores para el tratamiento eficiente de agua contaminada con compuestos orgánicos. En E. San Martin Martinez, M. A. Ramírez Salinas (Eds.). Avances de investigación en Nanociencias, Micro y Nanotecnologías. Barcelona, España: OmniaScience. 117-131. 


\section{Resumen}

Se sintetizaron hidróxidos dobles laminares de $\mathrm{ZnCuAl}$ con y sin la adición de dodecilsulfato de sodio (SDS) como agente modificante, del que ha sido reportado que, al ser integrado durante la síntesis en hidróxidos dobles laminares incrementa su eficiencia fotocatalítica, una vez calcinados. Los materiales sintetizados fueron caracterizados mediante las técnicas de difracción de rayos X, microscopía electrónica de barrido y espectroscopía IR y UV-Vis para determinar sus propiedades estructurales, morfológicas, químicas y ópticas, y posteriormente evaluados en la reacción de fotodegradación de fenol en presencia de luz UV. La estructura laminar característica del material se conservó en el material con SDS, pero se pudo observar un incremento del valor de energía de banda prohibida al material contendiendo este agente. En los resultados de reacción, el material modificado mostró una fotoactividad casi $35 \%$ superior al material sin modificar. Este incremento en la eficiencia fotocatalítica podría ser debido a una posible generación de radicales $\mathrm{SO}_{4}{ }^{\bullet-}$ a partir de la irradiación de los grupos sulfato del SDS, los cuales son altamente oxidantes en este tipo de reacciones de fotooxidación en fase acuosa.

Palabras clave: Fotodegradación; fotocatálisis; agua; hidróxidos dobles laminares; fenol. 


\section{Introducción}

Entre estos contaminantes orgánicos, el fenol y sus derivados son de los más recurrentes, ya que es empleado de manera intensiva en la industria farmacéutica como antiséptico, anestésico y desinfectante. Su ingesta es altamente tóxica y produce alteraciones en las paredes celulares y necrosis por coagulación, entre otros efectos, además de ser fácilmente absorbido a través de la piel, pudiendo ser letal a dosis de 1 gramo. Algunos de los síntomas que se presentan por su presencia en el organismo humano son náuseas, diarrea hemática, vómitos, dolor abdominal, barras blancas en la mucosa oral, en algunas ocasiones se pueden presentar quemaduras, convulsiones, coma, taquicardia, disritmias, hipotensión, hipotermia, acidosis metabólica, alteraciones pulmonares, aliento dulce, quemaduras cutáneas cuando se tienen niveles altos de la molécula y puede generar cáncer o incluso la muerte [1].

Entre los métodos para eliminar contaminantes orgánicos presentes en agua como el fenol y sus derivados, los procesos fotocatalíticos, consistentes en una serie de reacciones redox que se llevan a cabo en sistemas constituidos por materiales semiconductores (fotocatalizadores) y una fuente de luz radiante con energía similar o mayor al valor de energía de banda prohibida de los mismos, ha cobrado cada vez más importancia.

Los hidróxidos dobles laminares (HDL), también llamados hidrotalcitas [1], son compuestos basados en la estructura de la brucita $(\mathrm{MgO})$, en donde a través de una sustitución isomórfica parcial de iones metálicos divalentes como el magnesio $\left(\mathrm{M}^{2+}\right)$ por iones metálicos trivalentes, como el aluminio $\left(\mathrm{M}^{3+}\right)$, se generan láminas con carga positiva, la cual es compensada por la presencia de aniones en el espacio interlaminar (Figura 1).

La fórmula general de los hidróxidos dobles laminares puede ser expresada como:

$$
\left[\mathrm{M}^{2+}+\mathrm{x} \mathrm{M}^{3+}{ }_{1-\mathrm{x}}(\mathrm{OH})_{2 \mathrm{x}} \mathrm{A}_{\mathrm{m}^{-}} \cdot \mathrm{zH}_{2} \mathrm{O}\right][2]
$$

donde:

$\mathrm{M}^{2+} \mathrm{y} \mathrm{M}^{3+}$ son iones metálicos divalentes y trivalentes, $\mathrm{y}_{\mathrm{m}}$ - es un anión intercalado $\left(\mathrm{CO}_{3}^{2-}\right.$ el más común)

$\mathrm{Al}$ ser sometidos a tratamiento térmico, los hidróxidos dobles laminares sufren un proceso de deshidroxilación, convirtiendo los hidróxidos que los conforman 

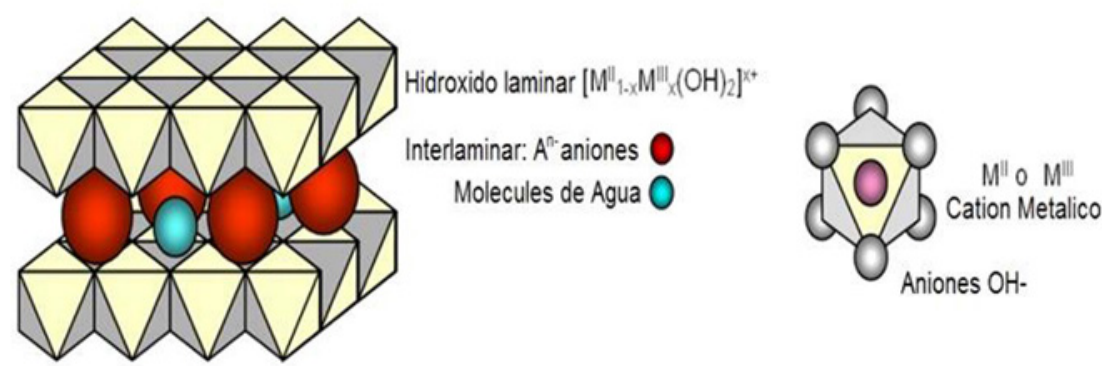

\section{Estructura del Hidróxido Doble Laminar}

Figura 1. Estructura de la brucita y de los hidróxidos dobles laminares.

en sus respectivos óxidos mixtos, los cuales han mostrado ser fotocatalizadores activos en reacciones de degradación de contaminantes orgánicos en medio acuoso [3-8]. Particularmente en el área de fotocatálisis se ha prestado especial atención a los hidróxidos dobles laminares de $\mathrm{ZnAl}$, debido a que a través de su calcinación es posible obtener óxido de zinc, que junto con el óxido de titanio se encuentran entre los fotocatalizadores mayormente reportados en la literatura [9-18], aunque otros sistemas en donde se incluye metales como Ni y Cu han sido estudiados obteniendo excelentes resultados [19].

Sin embargo, aunque los óxidos mixtos derivados de los hidróxidos dobles laminares han dado excelentes resultados, el proceso de calcinación involucra un gasto energético que incrementa los costos de los materiales, por lo cual se buscó la alternativa de emplear como fotocatalizadores para la degradación de contaminantes orgánicos en presencia de irradiación UV los hidróxidos dobles laminares sin calcinar, ya que aunque éstos no son materiales semiconductores, cuentan con una alta población de $\mathrm{OH}$ en su superficie y podrían propiciar la generación de radicales $\mathrm{OH} \bullet$ durante su irradiación con luz UV, los cuales son generados como parte del mecanismo de oxidación de compuestos orgánicos empleando procesos fotocatalíticos. Adicionalmente, trabajos previos [20] mostraron que, mediante la adición de dodecilsulfato de sodio como agente modificante en fotocatalizadores basados en hidróxidos dobles laminares de $\mathrm{ZnAl}$ calcinados, se lograba mejorar la actividad del fotocatalizador en la degradación fotocatalítica de fenol en presencia de luz visible. Con base en lo anterior, se decidió probar la eficiencia de hidróxidos dobles laminares de $\mathrm{ZnCuAl}$, modificados con la adición de dodecilsulfato de sodio (SDS) y sin calcinar como fotocatalizadores para la degradación de fenol en medio acuoso en presencia de luz UV. 


\section{Materiales y métodos}

Se realizó la síntesis de hidróxidos dobles laminares de $\mathrm{ZnAlCu}$ con y sin la adición de dodecilsulfato de sodio, empleando para ello el método de coprecipitación. Como reactivos de partida se emplearon nitratos de zinc, aluminio y cobre, con los cuales se preparó una solución, calculando una relación molar teórica de 2/1/1 para $\mathrm{Zn} / \mathrm{Cu} / \mathrm{Al}$. La solución obtenida se mantuvo a un valor constante de $\mathrm{pH}=9$ mediante la adición de urea al sistema reaccionante, manteniendo en agitación magnética durante 12 horas a una temperatura controlada de $90^{\circ} \mathrm{C}$, empleando para ello un sistema de reflujo. En el caso del material con adición de SDS, se procedió de la misma manera, pero a la solución reaccionante se le agregaron $10 \mathrm{~mL}$ de una solución de dodecilsulfato de sodio $(0,02 \mathrm{~mol}$ de $\mathrm{SDS} / \mathrm{L}$ ) durante la síntesis.

La cristalinidad de los materiales sintetizados se determinó mediante el análisis por difracción de Rayos X empleando para ello un difractómetro marca BrukerAXS, modelo D8-advance, el análisis morfológico se realizó por microscopía electrónica de barrido (SEM, por sus siglas en inglés) y el análisis de la composición elemental se realizó con un detector (JEOL, EX94400T4L11 Dry SD); los espectros de IR se obtuvieron mediante un equipo (Shimadzu, IRTracer-100). La medición de las propiedades ópticas se realizó empleando un equipo de espectroscopía UV-Vis (Agilent, CARY 100) con accesorio de reflectancia difusa, y los valores de energía de banda prohibida se calcularon mediante la ecuación de Kubelka-Munk.

La actividad fotocatalítica se evaluó en la reacción de fotodegradación de la molécula de fenol (en solución acuosa a concentración de 40 ppm), empleando un reactor de Pyrex de diseño especial, irradiando la mezcla reaccionante de la solución problema con $0.1 \mathrm{~g}$ del fotocatalizador con una lámpara de luz UV (254 $\left.\mathrm{nm}, 4400 \mu \mathrm{W} / \mathrm{cm}^{2}\right)$.

Se eligió al fenol como molécula orgánica a degradar ya que este es un contaminante frecuente en los efluentes industriales, que presenta con alta toxicidad para el ser humano y los ecosistemas, aun a bajas concentraciones, y por dificultad para ser degradado al tratarse de una molécula muy refractaria.

La detección de la disminución del contenido de fenol en la solución se realizó siguiendo la banda característica a $269 \mathrm{~nm}$ del espectro obtenido del análisis UV Vis, y cuantificando el fenol mediante los valores graficados de una curva 
de calibración con soluciones de fenol de concentración conocida previamente elaborada.

\section{Resultados}

Los espectros obtenidos del análisis de difracción de rayos $\mathrm{X}$ de los materiales $\mathrm{ZnCuAl}$ con y sin la adición de dodecilsulfato de sodio (SDS) se presentan en la Figura 2, donde se puede observar para ambos casos, la presencia de las señales características de los hidróxidos dobles laminares [21-23], con reflexiones a $2 \Theta$ $\approx 11.6,23.2^{\circ}, 34.5^{\circ}, 39.1^{\circ}, 46.7^{\circ}, 60.1^{\circ}$ y $61.43^{\circ}$, correspondientes a los planos (003), (006), (101), (015), (018), (110) y (113), respectivamente (PDF 38-0486).

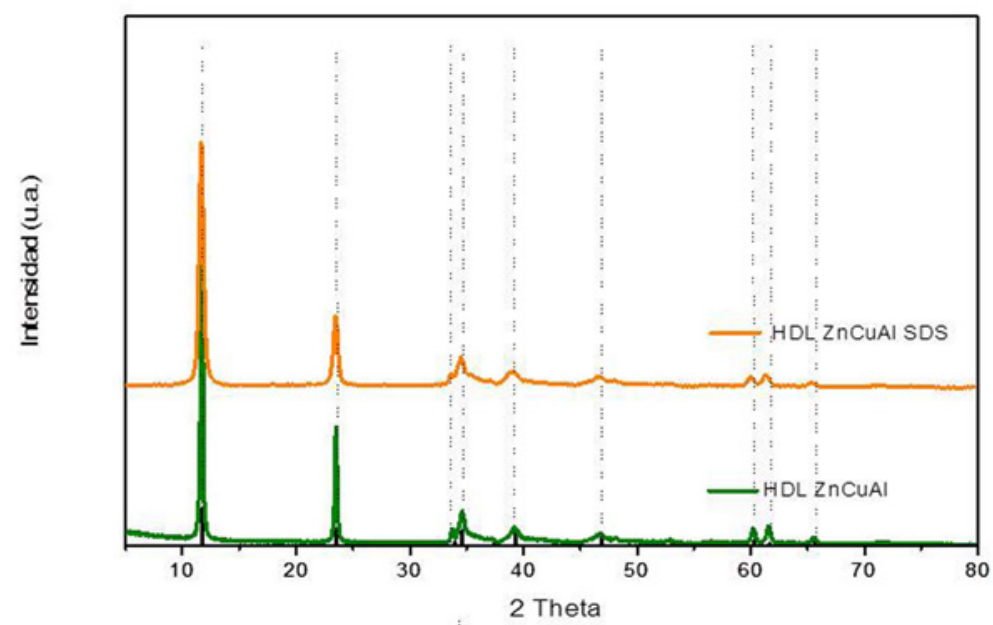

Figura 2. Patrones de difracción obtenidos para los $\mathrm{HDL} \mathrm{ZnCuAl}$ con y sin adición de SDS.

Respecto a los resultados del análisis por espectroscopía IR de las muestras se puede observar la presencia de una banda amplia e intensa alrededor de 3100 a $3600 \mathrm{~cm}^{-1}$, en la que se encentran englobadas señales correspondientes a vibraciones o estiramientos de diferentes enlaces: una banda observada entre 3100-3300 $\mathrm{cm}^{-1}$ relacionada con el enlace $\mathrm{C}-\mathrm{H}$; a $3330 \mathrm{~cm}^{-1}$ podemos localizar vibraciones de $\mathrm{O}-\mathrm{H}$ y entre $3300-3500 \mathrm{~cm}^{-1}$ la vibración de tensión del enlace $-\mathrm{H}-\mathrm{N}-$; la banda a $3500 \mathrm{~cm}^{-1}$ corresponde a los estiramientos de los grupos hidroxilo tanto de las láminas como del agua del espacio interlaminar.

Adicionalmente a esta banda, se puede identificar una banda de absorción a $1364 \mathrm{~cm}^{-1}$ pertenece a los carbonatos presentes en el espacio interlaminar y una 
banda en la región de 400-1000 $\mathrm{cm}^{-1}$, que está relacionada con las vibraciones de oxígeno [24]. Por otra parte, las bandas de espectro correspondientes a 1635 y $1385 \mathrm{~cm}^{-1}$ pertenecen a vibraciones de flexión de enlaces O-H [25] y finalmente, la banda de absorción a $465 \mathrm{~cm}^{-1}$, que es típica de las vibraciones de estiramiento reticular $v(\mathrm{Zn}-\mathrm{O})$ [26] (que corresponden también a picos atribuidos a modos de estiramiento M-O). Solamente en el caso de los materiales con SDS se puede apreciar una banda en la región de 1040 a $1060 \mathrm{~cm}^{-1}$, correspondiente a enlaces $\mathrm{S}=\mathrm{O}$, así como otra banda entre $1300-1340 \mathrm{~cm}^{-1}$ relacionada con enlaces $\mathrm{SO}_{2}$ (Figura 3).

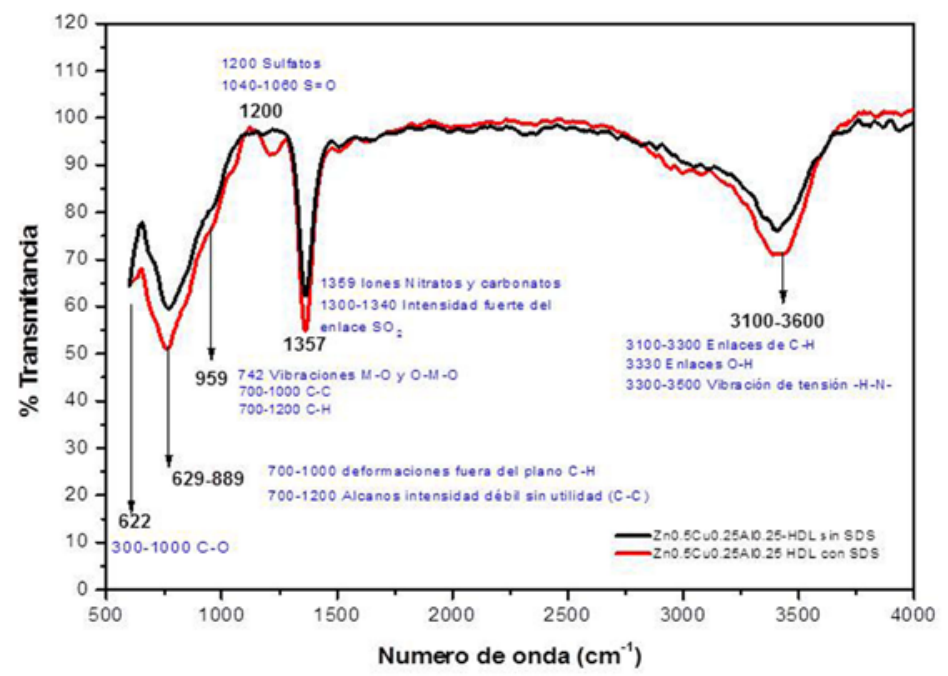

Figura 3. Espectros de IR de hidróxidos dobles laminares $\mathrm{ZnCuAl}$, con y sin adición de SDS.

Los valores de energía de banda prohibida de los materiales modificados con SDS se calcularon empleando la ecuación de Kubelka Munk, empleando para ello los valores de reflectancia obtenidos mediante la medición por espectroscopía UV, y realizando la interpolación lineal de éstos a lo largo del borde de absorción. De los resultados obtenidos de este cálculo se pudo apreciar que mediante la integración de SDS al material se produjo un incremento en el valor del ancho de banda prohibida, de un valor de $2.73 \mathrm{eV}$ a $3.19 \mathrm{eV}$, para los $\mathrm{HDL}$ de $\mathrm{ZnCuAl}$ y $\mathrm{ZnCuAl} \mathrm{SDS}$, respectivamente.

Respecto a la morfología de los hidróxidos dobles laminares $\mathrm{ZnAlCu}$ modificados con SDS, las micrografías obtenidas mediante el análisis por microscopía electrónica de barrido muestran la presencia de partículas conglomeradas en forma de plaquetas hexagonales que es característica este tipo de materiales [27-30] 


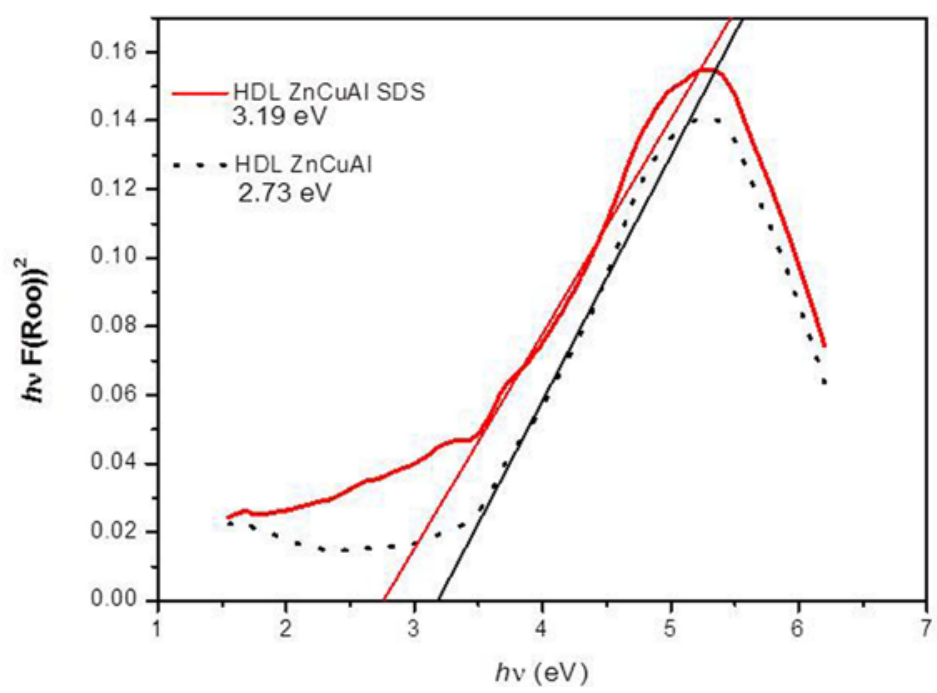

Figura 4. Determinación de los valores de energía de banda prohibida para los HDL $\mathrm{ZnAl}$ y $\mathrm{ZnCuAl}$ sintetizados.

con bordes no muy definidos (Figura 4), lo cual ha sido reportado por algunos autores para HDLs modificados mediante la adición de un compuesto orgánico durante su síntesis [31].
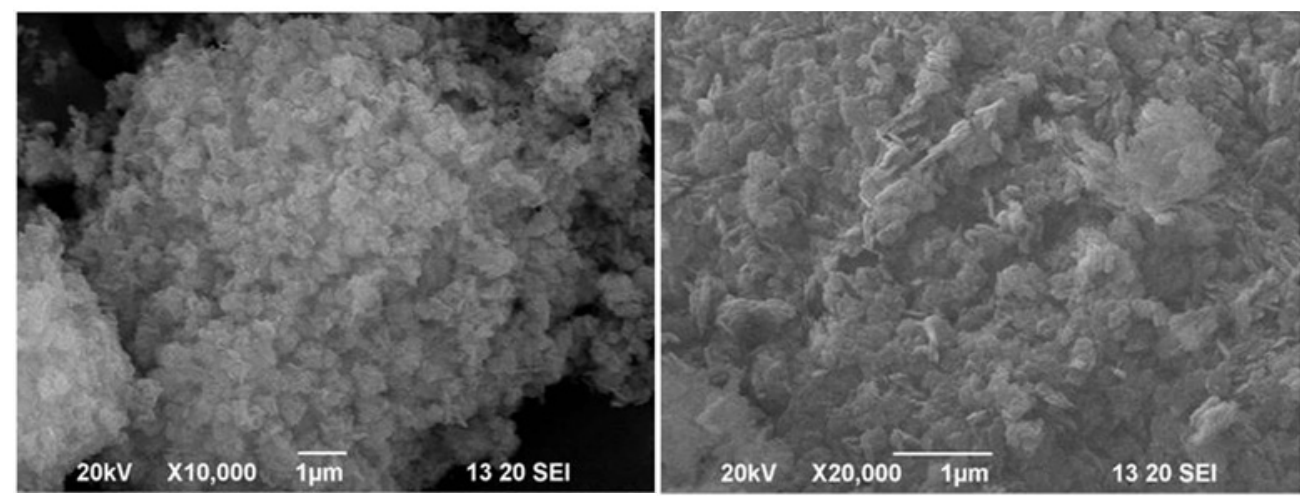

Figura 5. Imágenes obtenidas por microscopía electrónica de barrido del HDL ZnAlCu SDS.

Los resultados obtenidos de la fotodegradación de fenol con los HDL $\mathrm{ZnCuAl}$ con y sin adición de SDS se presentan en la Figura 6, donde podemos observar que a pesar de tener un valor de energía de banda prohibida (band gap) mayor, el material con SDS mostró un incrementó en la actividad fotocatalítica respecto al material sin modificar, con un $80 \%$ vs $60 \%$ de degradación de fenol, respectivamente. 


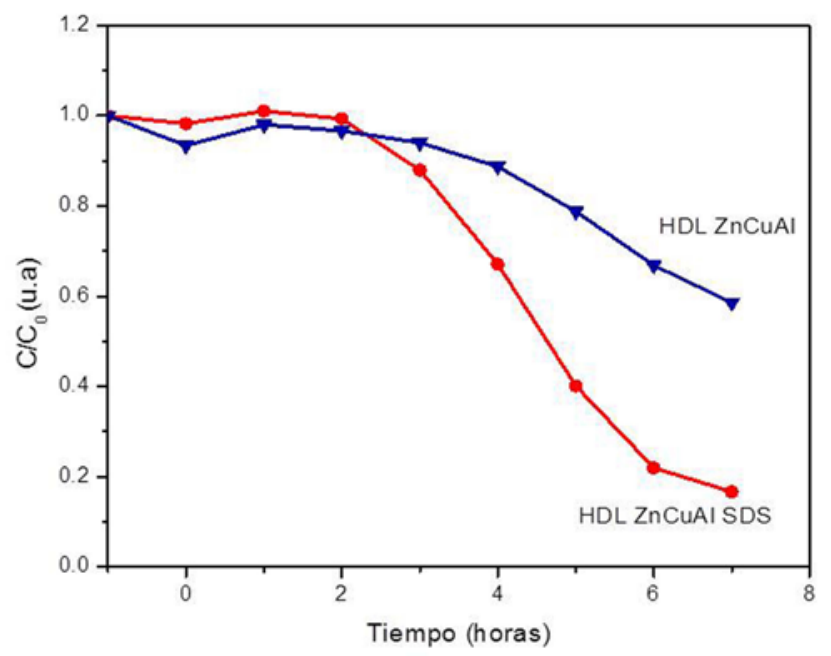

Figura 6. Fotodegradación de la molécula de fenol en presencia de luz UV empleando hidróxidos dobles laminares $\mathrm{ZnCuAl}$ con y sin la incorporación de SDS.

Este resultado podría ser explicado con base en las reacciones del proceso fotocatalítico, donde un material semiconductor al tener una interacción con una fuente de irradiación, produce los denominados pares electrón-hueco $\left(e^{-} / b^{+}\right)$en la superficie del mismo; este par $\left(e^{-} / b^{+}\right)$en presencia de la molécula de agua genera a su vez radicales capaces de llevar a cabo las reacciones de fotooxidación de compuestos orgánicos.

Como se mencionó anteriormente, en reacciones en fase acuosa ocurre una adsorción espontánea en el semiconductor y, dependiendo del potencial redox (o nivel de energía) de cada adsorbato, se efectúa una transferencia de electrones $(e)$ hacia las moléculas aceptoras; por otro lado, el hueco positivo $\left(b^{+}\right)$es transferido a una molécula donadora, como se describe en las siguientes ecuaciones:

$$
\begin{array}{ll}
\text { Ec. } 1 & b v+S C \rightarrow e^{-}+b^{+} \\
\text {Ec. } 2 & A(a d s)+e^{-} \rightarrow A(a d s)^{-} \\
\text {Ec. } 3 & D(a d s)+b^{+} \rightarrow D(a d s)^{+}
\end{array}
$$

Por ello, podemos decir que la excitación fotónica del material semiconductor es el primer paso de la activación de todo el sistema de reacciones involucradas en un sistema fotocatalítico. Por otra parte, el radical $\mathrm{OH}^{\bullet}$ formado durante las reacciones del proceso fotocatalítico es conocido como especie oxidante muy reactiva, capaz de degradar moléculas de compuestos orgánicos como el fenol, las cuales como ya se mencionó, son absorbidas durante el proceso en la superficie 
del fotocatalizador (en este caso $\mathrm{LDH} \mathrm{ZnCuAl}$ ). La fotodegradación entonces se efectúa mediante un mecanismo complejo, el cual se esquematiza en la Figura 7.

En el caso del fotocatalizador $\mathrm{HDL} \mathrm{ZnCuAl} \mathrm{SDS}$, la presencia de grupos sulfatos provenientes de la molécula del dodecilsulfato de sodio (SDS) podrían generar, en presencia de la irradiación de luz UV, radicales $\mathrm{SO}_{4}{ }^{-}$, los cuales han sido reportados con un alto potencial de oxidación, incluso superior al presentado por los radicales $\mathrm{OH} \bullet$ involucrados en el proceso fotocatalítico tradicional, y al intervenir éstos en las reacciones de este proceso, incrementaría la velocidad de degradación de la molécula de fenol, como se pudo ver en los resultados de evaluación.

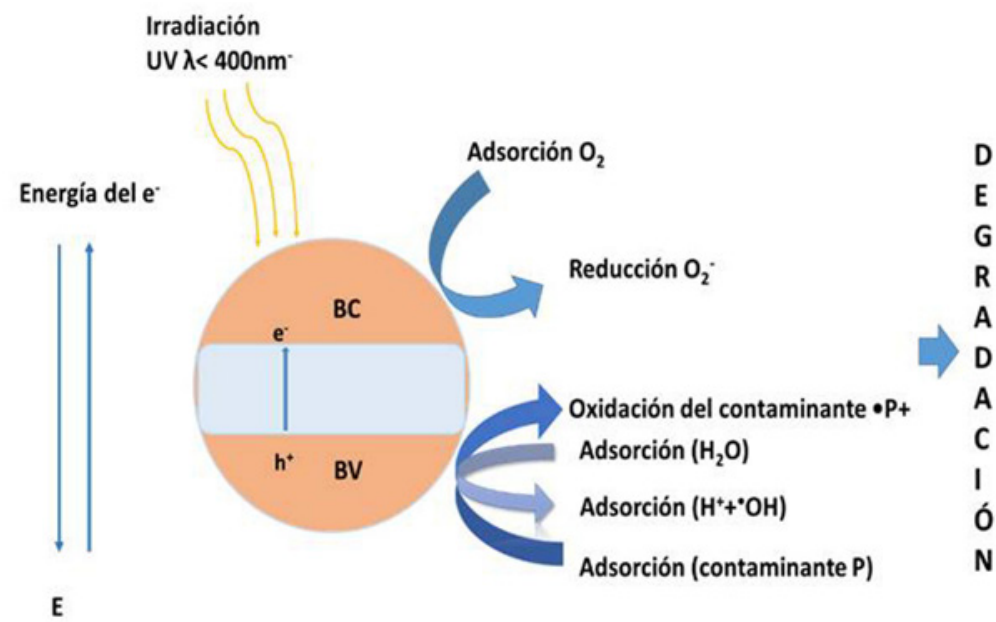

Figura 7. Esquema del sistema de reacciones involucradas durante el proceso fotocatalítico de manera tradicional.

Las reacciones propuestas para el mecanismo propuesto se presentan a continuación:

Ec $1 \quad \mathrm{ZnAl-SDS}+b v \rightarrow b^{+} B V+e^{-} B C$

$\mathrm{Ec} 2$ e- $\mathrm{BC}+\mathrm{O}_{2} \rightarrow \mathrm{O}_{2}^{-}$

$\mathrm{Ec} 3(a) \quad b^{+} \mathrm{BV}+\mathrm{H}_{2} \mathrm{O} \rightarrow \mathrm{OH}^{\cdot}+\mathrm{H}^{+}$

$\mathrm{Ec} 3$ (b) $b^{+} \mathrm{BV}+\mathrm{SO}_{4} \rightarrow \mathrm{SO}_{4}{ }^{\cdot-}$

$\mathrm{Ec} 4 \quad\left(\mathrm{SO}_{4}^{--}+\mathrm{OH}\right)+\mathrm{Fenol} \rightarrow$ degradación

\section{Conclusión}

Se pudo constatar que la integración de dodecilsulfato de sodio (SDS) como agente modificante incrementa la actividad fotocatalítica de los hidróxidos dobles 
laminares para reacciones de degradación de compuestos orgánicos como el fenol en medio acuoso, empleando como fuente de irradiación luz UV de baja intensidad. Este comportamiento podría ser explicado mediante una posible generación, a partir de los sulfatos contenidos en la molecula del SDS, de radicales $\mathrm{SO}_{4}{ }^{\bullet}$, ya que estos radicales han sido reportados con un alto potencial de oxidación, mayor al de los radicales $\mathrm{OH} \bullet$-involucrados en la mayoría de los casos para este tipo de reacciones de oxidación fotocatalítica.

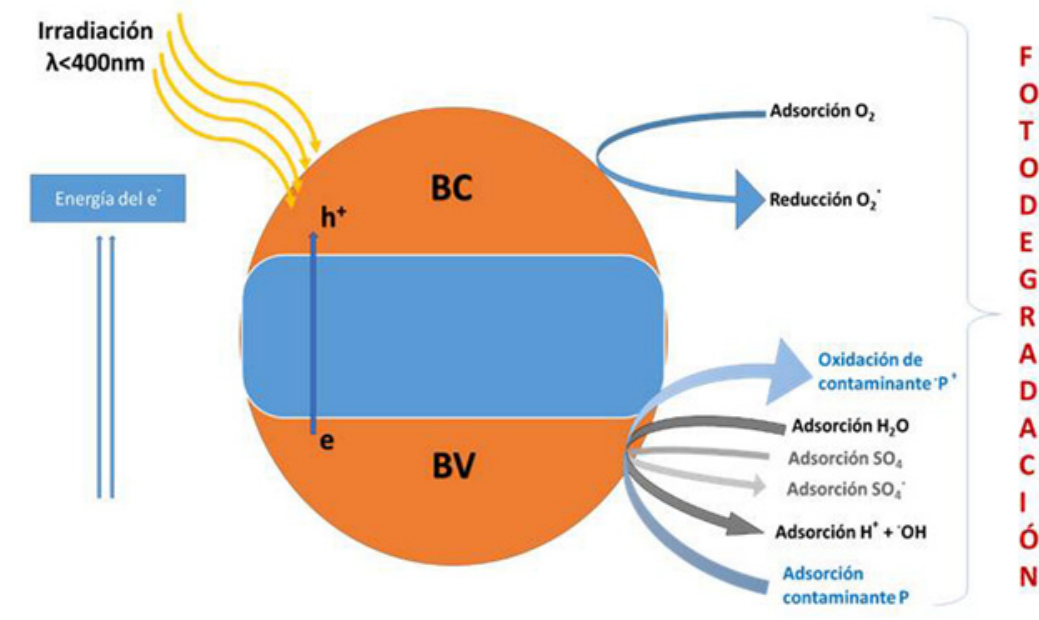

Figura 8. Esquema del sistema de reacciones propuesto, incorporando la generación de radicales $\mathrm{SO}_{4} \bullet$ en el proceso fotocatalítico.

\section{Agradecimientos}

Los autores agradecen el apoyo económico de los proyectos SIP 20201317, 20200352 y CONACyT CB 285711. G. Romero agradece a CONACyT y BEIFI por las becas otorgadas. y Y. Jiménez agradece a CONACyT por el apoyo de la beca de estancia post doctoral.

\section{Referencias}

1. Carrasco Jiménez, M. S., de Paz Cruz, J. A. (s.f.). Tratado de emergencias médicas (II). España: Arán Ediciones.

2. Cavani, F. Trifiró, F. Vaccari, A. (1991). Hydrotalcite-type anionic clays: Preparation, properties and applications. Catal. Today, 11, 173-301. https://doi.org/10.1016/09205861(91)80068-K 
3. Tzompantzi, F. Mendoza, G., Rico, J. L., Mantilla, A. (2014). Enhanced photoactivity for the phenol mineralization on ZnAlLa mixed oxides prepared from calcined LDHs. Catal. Today, 220, 56-60. https://doi.org/10.1016/j.cattod.2013.07.014

4. Mantilla, A. Tzompantzi, F, Fernández, J. L., Díaz-Góngora, J. A. L., Gómez, R. (2010). Photodegradation of phenol and cresol in aqueous medium by using $\mathrm{Zn} / \mathrm{Al}+\mathrm{Fe}$ mixed oxides obtained from layered double hydroxides materials. Catal. Today, 150, 353-357. https://doi.org/10.1016/j.cattod.2009.11.006

5. Xiang, X., Xie, L., Li, Z., \& Li, F. (2013). Ternary MgO/ZnO/In2O3 heterostructured photocatalysts derived from a layered precursor and visible-light-induced photocatalytic activity. Chem. Eng. J., 221, 222-229. https://doi.org/10.1016/j.cej.2013.02.030

6. Seftel, E. M., Popovici, E., Mertens, M. Cool, P., \& Vansant, E. F. (2008). Sn ${ }^{\mathrm{IV}}$-containing layered double hydroxides as precursors for nano-sized $\mathrm{ZnO} / \mathrm{SnO} 2$ photocatalysts $A p p l$. Catal., B 84, 699-705. https://doi.org/10.1016/j.apcatb.2008.06.006

7. Tzompantzi, F., Mantilla, A., Bañuelos, F., Fernández, J., \& Gómez, R. (2011). Improved Photocatalytic Degradation of Phenolic Compounds With ZnAl Mixed Oxides Obtained from LDH Materials. Top. Catal, 54, 257-263. https://doi.org/10.1007/s11244-011-9656-3

8. Mendoza-Damián, G., Tzompantzi, F., Mantilla, A., Barrera, A., Lartundo-Rojas, L., \& Hazard, J. (2013). Photocatalytic degradation of 2,4-dichlorophenol with MgAlTi mixed oxides catalysts obtained from layered double hydroxides. Mater, 263, 67-72. https:// doi.org/10.1016/j.jhazmat.2013.09.047

9. Patzko, A., Kun, R., Hornok, V., Dekany, I., Engelhardt, T., \& Schall, N. (2005). ZnAl-layer double hydroxides as photocatalysts for oxidation of phenol in aqueous solution. Colloids and Surfaces A: Physicochem. Eng. Aspects, 265, 64-72. https://doi.org/10.1016/j. colsurfa.2005.01.039

10. Ahmed, N., Shibata, Y., Taniguchi, T., \& Izumi, Y. (2011). Photocatalytic conversion of carbon dioxide into methanol using zinc-copper-M(III) ( $\mathrm{M}=$ aluminum, gallium) layered double hydroxides. J. Catal., 279, 123-135. https://doi.org/10.1016/j. jcat.2011.01.004

11. Martín del Campo, E., Valente, J.S. Pavón, T. Romero, R. Mantilla, A. Natividad, R. (2011). 4-Chlorophenol Oxidation Photocatalyzed by a Calcined Mg-Al-Zn Layered Double Hydroxide in a Co-current Downflow Bubble Column. Ind. Eng. Chem. Res., 50, 11544-11552. https://doi.org/10.1021/ie200412p

12. Mantilla, A., Tzompantzi, F., Fernández, J. L., Díaz-Góngora, J. A. I., Mendoza, G., \& Gómez, R. (2009). Photodegradation of 2,4-dichlorophenoxyacetic acid using ZnAlFe layered double hydroxides as photocatalysts. Catal. Today, 148, 119-123. https://doi. org/10.1016/j.cattod.2009.02.036 
13. Seftel, E. M., Popovici, E., Mertens, M., De Witte, K., Van Tendeloo, G., Cool, P., \& Vansant, E. F. (2008). Zn-Al layered double hydroxides: Synthesis, characterization and photocatalytic application. Microporous Mesoporous Mater., 113, 296-304. https://doi. org/10.1016/j.micromeso.2007.11.029

14. Parida, M., Baliarsingh, N., Sairam Patra, B., Das, J., \& Mol, J. (2007). Copperphthalocyanine immobilized $\mathrm{Zn} / \mathrm{Al} \mathrm{LDH}$ as photocatalyst under solar radiation for decolorization of methylene blue. Catal. A: Chem. 267, 202-208. https://doi.org/10.1016/j. molcata.2006.11.035

15. Parida, K. M., \&. Mohapatra, L. (2012). Recent progress in the development of carbonate-intercalated $\mathrm{Zn} / \mathrm{Cr} \mathrm{LDH}$ as a novel photocatalyst for hydrogen evolution aimed at the utilization of solar light. Dalton Trans., 41, 1173-1178. https://doi.org/10.1039/ C1DT10957J

16. Mohapatra, L., \& Parida, K. M. (2012). Zn-Cr layered double hydroxide: Visible light responsive photocatalyst for photocatalytic degradation of organic pollutants. Sep. Purif. Technol., 91, 73-80. https://doi.org/10.1016/j.seppur.2011.10.028

17. Lam, S. M., Sin, J., Abdullah, A. Z., \& Mohamed, A. R. (2012). Degradation of wastewaters containing organic dyes photocatalysed by zinc oxide: a review. Desalin. Water Treat., 41, 131-169. https://doi.org/10.1080/19443994.2012.664698

18. Rezaei, M., \& Habibi-Yangjeh, A. (2013). Microwave-assisted preparation of Ce-doped $\mathrm{ZnO}$ nanostructures as an efficient photocatalyst. Mater. Lett., 110, 53-56. https://doi. org/10.1016/j.matlet.2013.07.120

19. Carja G., Dartu L., Okada K., Fortunato E. (2013). Nanoparticles of copper oxide on layered double hydroxides and the derived solid solutions as wide spectrum active nano-photocatalysts. Chem. Eng. J., 222, 60-66. https://doi.org/10.1016/j.cej.2013.02.039

20. Romero Ortíz, G. (2015). Desarrollo de fotocatalizadores basados en bidrotalcitas modificadas para la fotodegradación de contaminantes en medio acuoso. Tesis para obtener el Grado de Maestría en Tecnología Avanzada, CICATA Legaria, Instituto Politécnico Nacional.

21. Shu, Z., Guo, Q., Chen, Y., Zhou, J., Guo, W., \& Cao, Y. (2017). Accelerated sorption of boron from aqueous solution by few-layer hydrotalcite nanosheets. Applied Clay Science, 149, 13-19. https://doi.org/10.1016/j.clay.2017.09.003

22. Smoláková, L., Dubnová, 1., Kocík, J., Endres, J., Daniš, S., Priecel, P., \& Čapek, L. (2018). In-situ characterization of the thermal treatment of $\mathrm{Zn}-\mathrm{Al}$ hydrotalcites with respect to the formation of $\mathrm{Zn} / \mathrm{Al}$ mixed oxide active in aldol condensation of furfural. Applied Clay Science, 157, 8-18. https://doi.org/10.1016/j.clay.2018.02.024

23. Ch. Yang, L. L. J. of Coll. and Interf. Sc. (2016) 115-120. 
24. Janusz Nowicki, J. L. (2016). Transesterification of rapeseed oil to biodiesel over Zr-dopped MgAl hydrotalcites. Appl. Catal. A: General, 524, 17-24. https://doi. org/10.1016/j.apcata.2016.05.015

25. Liang, X. Yang, X. Gao, G. Li, C., Yuanyuan, L., Zhang, W. et al. (2016). Performance and mechanism of $\mathrm{CuO} / \mathrm{CuZnAl}$ hydrotalcites- $\mathrm{ZnO}$ for photocatalytic selective oxidation of gaseous methanol to methyl formate at ambient temperature J. of Catal. 339, 68-76. https://doi.org/10.1016/j.jcat.2016.03.033

26. El-Nahhal, I. M., Elmanama, A. A., El Ashgar, N. M., Amara, N., Selmane, M., \& Chehimi, M. M. (2017). Stabilization of nano-structured $\mathrm{ZnO}$ particles onto the surface of cotton fibers using different surfactants and their antimicrobial activity. Ultrasonics Sonochemistry, 38, 478-487. https://doi.org/10.1016/j.ultsonch.2017.03.050

27. Zhang, F., Du, N., Song, S., Liu, J., \& Hou, W. (2013). Mechano-hydrothermal synthesis of Mg2Al-NO3 layered double hydroxides. J. Solid State Chem. 206, 45-50. https://doi. org/10.1016/j.jssc.2013.07.030

28. Zhang, F., Du, N., Li, H., Liu, J., \& Hou, W. (2014). Synthesis of Mg-Al-Fe-NO3 layered double hydroxides via a mechano-hydrothermal route. J. Solid State Sci., 32, 41-47. https://doi.org/10.1016/j.solidstatesciences.2014.03.012

29. Abelló, S., Medina, F., Tichit, D., Pérez-Ramírez, J., Rodrígueza, X., Sueirasa, J. E., Salagrea, P., \& Cesterosa, Y. (2005). Study of alkaline-doping agents on the performance of reconstructed $\mathrm{Mg}-\mathrm{Al}$ hydrotalcites in aldol condensations. Appl. Catal. A: Gen., 281, 191-198. https://doi.org/10.1016/j.apcata.2004.11.037

30. Greenwell, H. C., Holliman, P. J., Jones, W., \& Velasco, B. V. (2006). Studies of the effects of synthetic procedure on base catalysis using hydroxide-intercalated layer double hydroxides. Catal. Today 114, 397-402. https://doi.org/10.1016/j.cattod.2006.02.035

31. Costa, F. R., Leuteritz, A., Wagenknecht, U., Jehnichen, D., Häußler, L., \& Heinrich, G. (2008). Intercalation of $\mathrm{Mg}-\mathrm{Al}$ layered double hydroxide by anionic surfactants: Preparation and characterization. Applied Clay Science, 38, 153-164. https://doi.org/10.1016/j. clay.2007.03.006 